\title{
A. Alcouffe \\ M. ENJALBERT \\ G. MURATET \\ Méthodes de résolution du problème de transport et de production d'une entreprise à établissements multiples en présence de coûts fixes
}

Revue française d'automatique, d'informatique et de recherche opérationnelle. Recherche opérationnelle, tome 9, $\mathrm{n}^{\circ} \mathrm{V} 3$ (1975), p. 41-55.

$<$ http://www.numdam.org/item?id=RO_1975_9_3_41_0>

(C) AFCET, 1975, tous droits réservés.

L'accès aux archives de la revue « Revue française d'automatique, d'informatique et de recherche opérationnelle. Recherche opérationnelle » implique l'accord avec les conditions générales d'utilisation (http://www.numdam.org/ legal.php). Toute utilisation commerciale ou impression systématique est constitutive d'une infraction pénale. Toute copie ou impression de ce fichier doit contenir la présente mention de copyright.

\section{Numdam}




\title{
MÉTHODES DE RÉSOLUTION DU PROBLĖME DE TRANSPORT ET DE PRODUCTION D'UNE ENTREPRISE A ÉTABLISSEMENTS MULTIPLES EN PRÉSENCE DE COÛTS FIXES (*)
}

\author{
par A. Alcouffe $\left({ }^{1}\right)$, M. Enjalbert $\left({ }^{2}\right)$ et G. Muratet $\left({ }^{3}\right)$
}

\begin{abstract}
Résumé. - Cet article étudie le problème d'une entreprise qui doit satisfaire une demande donnée émanant de clients disséminés et qui doit choisir les lieux d'implantation des établissements où elle réalisera sa production. Le coût de production comprend une charge fixe par établissement construit et un cô̂t proportionel à la quantité produite. Le coût de transport entre un établissement et un client est proportionnel aux quantités transportées. L'entreprise cherche à ininimer les coûts de transport et de production.

Cet article décrit comment tirer des propriétés des coûts une procédure de résolution du problème. Il présente également une méthode heuristique dont le principe consiste dans une simulation de la concurrence entre entreprises indépendantes.
\end{abstract}

\section{INTRODUCTION}

De nombreuses entreprises dont les clients sont géographiquement dispersés répartissent leur production entre plusieurs établissements dont chacun se caractérise par une localisation et une fonction de coût particulières. Aussi pour assurer l'approvisionnement de leurs clients au moindre coût doivent-elles considérer à la fois le niveau de production de chaque établissement et le couplage établissement-client. Elles ont donc à résoudre simultanément un problème de transport et un problème de détermination optimale des niveaux de production.

La résolution du problème dépend étroitement des propriétés mathématiques des fonctions de coût. La littérature présente des solutions dans quelques cas.

(*) Reçu août 1974.

(1) U.E.R. de Sciences Économiques, Université des Sciences Sociales, Toulouse.

(2) Institut du Génıe Chimıque, Toulouse.

$\left({ }^{3}\right)$ Institut du Génie Chimique, Toulouse.

Revue Française d'Automatique, Informatique et Recherche Opérationnelle $\mathrm{n}^{\circ}$ oct. 1975, V-3. 
10 Patinkin [1] et Leontief [2] ont étudié le cas d'une entreprise à établissements multiples qui envisage la meilleure répartition possible de la production connaissant le niveau de la demande globale mais qui ne prend pas en considération les coûts de transport. V. L. Smith [9] qui a poursuivi leur analyse soutient que des solutions empiriques ingénieuses avaient déjà été apportées à ce problème au $\mathrm{XIX}^{\mathrm{e}}$ siècle par les entreprises produisant de l'énergie électrique.

$2^{\circ}$ Hadley [3] et Vaszony [4] ont montré comment l'algorithme de transport (Hitchcock-Koopmans) [5] pouvait être utilisé dans le cas où le coût de production moyen était indépendant du niveau de la production dans chaque établissement.

$3^{\circ}$ J. F. Sharp, J. C. Snyder et J. H. Greene [6] ont étudié le cas où les coûts de transport (entre chaque couple établissement-client) sont linéaires et où les coûts de production sont caractérisés par un coût marginal constamment croissant. Cette hypothèse de convexité des coûts de production leur permet d'établir, à partir des conditions de Kuhn et Tucker et du dual du problème de transport, un algorithme de résolution du problème de transport et de production dans les conditions particulières envisagées. Cette approche néglige un aspect essentiel du problème tel qu'il se présente effectivement du point de vue économique : en effet l'hypothèse de convexité des fonctions de coût interdit de prendre en compte des coûts fixes.

$4^{\circ}$ W. M. Hirsch, A. J. Hoffman et G. B. Dantzig [7] ont étudié le problème des charges fixes associées à des coûts proportionnels indépendants du niveau d'activité en envisageant un ensemble de contraintes linéaires plus général que celui que l'on rencontre dans le problème du transport. Ils ont montré que la solution du problème se trouve en un point extrême de l'espace des contraintes et que quand les coûts fixes sont égaux, il existe un programme linéaire associé qui permet de calculer l'optimum. Ils n'ont pas proposé d'algorithme dans le cas où les coûts fixes sont quelconques.

L'article suivant étudie la minimation des coûts de production et de transport d'une entreprise qui envisage de créer plusieurs établissements et qui connait le niveau de la demande et la localisation de ses différents clients. A l'approvisionnement des clients correspondent des coûts fixes et des coûts proportionnels.

\section{FORMULATION DU PROBLÈME}

\section{Soient :}

$N$, le nombre des clients;

$D_{j}$, la demande du client $j, j=1,2, \ldots, N$; 
$M$, le nombre des établissements;

$q_{i}$, la production de l'établissement $i, i=1,2, \ldots, M$;

$d_{i j}$, la quantité du produit livré par l'établissement $i$ au client $j$;

$t_{i i}$, le coût de transport d'une unité du produit de l'établissement $i$ au client $j\left(t_{i j}\right.$ est indépendant de la valeur de $\left.d_{i j}\right)$.

Le coût total de production dans l'établissement $i$ est égal à :

$$
C_{i}=a_{i} q_{i}+\delta_{i} b_{i},
$$

où $b_{i}$ représente les charges fixes imputées à la période envisagée,

$$
\delta_{i}=(0,1), \quad \delta_{i}=0 \Rightarrow q_{i}=0 .
$$

Le problème consiste à trouver les $\delta_{i}$, $q_{i}$ et $d_{i j}$ qui miniment le coût de l'approvisionnement des clients, compte tenu des contraintes imposées par la satisfaction des demandes :

$$
\operatorname{MIN} C_{T}=\operatorname{MIN} \sum_{i=1}^{M}\left[\left(a_{i} q_{i}+\delta_{i} b_{i}\right)+\sum_{i=1}^{M} \sum_{j=1}^{N} t_{i j} d_{i j}\right],
$$

sous les contraintes :

$$
\sum_{i=1}^{M} d_{i j}=D_{j}, \quad \forall j, \quad j=1,2, \ldots, N .
$$

Le non-gaspillage de la production impose que toute production réalisée soit livrée de sorte que :

$$
\sum_{j=1}^{N} d_{i j}=q_{i}, \quad \forall i, \quad i=1,2, \ldots, M .
$$

Et le coût à minimer s'écrit :

$$
\operatorname{MIN} C_{T}=\operatorname{MIN}\left[\sum_{i=1}^{M} \delta_{i} b_{i}+\sum_{i=1}^{M} \sum_{j=1}^{N}\left(a_{i}+t_{i j}\right) d_{i j}\right] .
$$

Pour simplifier l'écriture du problème, posons :

Il vient :

$$
a_{i}+t_{i j}=u_{i j}
$$

$$
\operatorname{MIN} C_{T}=\operatorname{MIN}\left[\sum_{i=1}^{M} \delta_{i} b_{i}+\sum_{i=1}^{M} \sum_{j=1}^{N} u_{i j} d_{i j}\right] .
$$

$n^{\circ}$ octobre 1975, V-3. 
Le problème apparaît alors comme un problème à décisions séquentielles dans lequel il faut choisir, dans un premier temps, les établissements actifs $\left(\delta_{i}=1\right)$ puis, dans un deuxième temps, fixer les niveaux de production des établissements retenus et les coupler avec les clients $\left(d_{i j}\right)$. Il est, donc, possible de lui appliquer le principe de la programmation dynamique et de l'écrire :

$$
\underset{\delta_{i}}{\operatorname{MIN}}\left[\sum_{i=1}^{M} \delta_{i} b_{i}+\underset{d_{i j}}{\operatorname{Min}} \sum_{i \in I} \sum_{j=1}^{N} u_{i j} d_{i j}\right],
$$

sous les contraintes :

$$
\sum_{i=1}^{M} d_{i j}=D_{j}
$$

où $I$ représente l'ensemble des établissements susceptibles de produire $\left(\delta_{i}=1\right)$.

Sous cette dernière forme, le problème

$$
\underset{d_{i j}}{\operatorname{Min}} \sum_{i \in I}^{M} \sum_{j=1}^{N} u_{i j} d_{i j}
$$

est un problème de transport classique dans lequel les coûts de production proportionnels jouent un rôle identique à celui des coûts unitaires de transport $\left(u_{i j}=a_{i}+t_{i j}\right)$. L'absence de contraintes liées à la capacité de production des établissements facilite la détermination de ce minimum. En effet, chaque client est approvisionné par un seul établissement, celui qui présente le coût $u_{i j}$ minimal. Le terme correspondant aux coûts proportionnels s'écrit alors :

$$
\sum_{j=1}^{N} \operatorname{Min}_{i \in I} u_{i j} D_{j}
$$

Le problème se présente, alors sous la forme :

$$
\begin{gathered}
\underset{\delta_{i}}{\operatorname{MIN}}\left[\sum_{i=1}^{N} \delta_{i} b_{i}+\sum_{j=1}^{M}\left(\operatorname{Min}_{i \in I} u_{i j}\right) D_{j}\right] \\
\left(i \in I \Leftrightarrow \hat{\delta}_{i}=1 ; I \neq \varnothing\right) .
\end{gathered}
$$

Nous avons écarté la programmation dynamique qui devient inefficace dès que le nombre d'établissements ou de clients dépasse 4 ou 5 [8]. Nous avons écarté également la programmation linéaire en nombres entiers car elle demande un grand volume de mémoire de calculateur pour enregistrer les différentes contraintes.

Nous présentons ici deux méthodes originales qui utilisent les propriétés intrinsèques du problème. 


\section{PREMIÈRE MÉTHODE}

Pour résoudre le problème qui vient d'être formulé, il est possible d'envisager successivement les différentes combinaisons d'établissements actifs, $\delta_{1}=1$, qu'il est possible de former, puis de calculer pour chacune, la répartition optimale des productions. Lorsque toutes les combinaisons ont été étudiées, celle qui donne le coût total minimal est l'optimum cherché.

Cette méthode, si elle conduit de manière certaine à l'optimum présente l'inconvénient de nous obliger à envisager un grand nombre de cas (soit $2^{M}-1$ s'il y a $M$ établissements). Dans le but de réduire le nombre des cas à étudier, nous avons cherché à exploiter certaines propriétés des coûts minimaux associés aux diverses combinaisons d'établissements.

\section{Propriété des coûts minimaux}

Considérons un ensemble d'établissements actifs $E_{0}$. Il lui correspond un coût minimal $C_{0}$ qui se décompose en un terme $F_{0}$ qui correspond aux coûts fixes et un terme $P_{0}$ qui correspond aux coûts proportionnels :

$$
\begin{gathered}
C_{0}=F_{0}+P_{0}, \\
F_{0}=\sum_{i \in E_{0}} b_{i}, \quad P_{0}=\sum_{j=1}^{N}\left(\operatorname{Min}_{i \in E_{0}} u_{i j}\right) D_{j} .
\end{gathered}
$$

Parmi les établissements de l'ensemble $E_{0}$, nous allons maintenant former deux sous-ensembles disjoints, $E_{1}$ et $E_{2}$ que nous allons successivement exclure. Soient $C_{1}$ et $C_{2}$ les coûts minimaux obtenus quand le sous-ensemble $E_{1}$, puis $E_{2}$, ne produit pas et $C_{12}$ le coût minimal lorsque ni $E_{1}$ ni $E_{2}$ ne produisent. Ces différents coûts s'expriment par les relations :

$$
\begin{gathered}
C_{1}=F_{0}-F_{1}+P_{1}, \\
F_{1}=\sum_{i \in E_{1}} b_{i}, \quad P_{1}=\sum_{j=1}^{N}\left(\underset{i \in \complement_{E_{0}} E_{1}}{\operatorname{Min}} u_{i j}\right) D_{j}, \\
C_{2}=F_{0}-F_{2}+P_{2}, \\
F_{2}=\sum_{i \in E_{2}} b_{i}, \quad P_{2}=\sum_{j=1}^{N}\left(\underset{i \in \prod_{E_{0}} E_{2}}{\operatorname{Min}} u_{i_{j}}\right) D_{j}, \\
C_{12}=F_{0}-F_{1}-F_{2}+P_{12}, \\
\left.P_{12}=\sum_{j=1}^{N} \underset{i \in \complement_{E_{0}} E_{1} \cup E_{2}}{\operatorname{Min}} u_{i j}\right) D_{j} .
\end{gathered}
$$

$\mathrm{n}^{\circ}$ octobre $1975, \mathrm{~V}-3$. 
La propriété que nous allons exploiter pour déterminer rapidement l'ensemble optimal d'établissements actifs se traduit de la manière suivante :

Si le coût $C_{1}$ ( $E_{1}$ exclu) est supérieur au coût $C_{0}$ (aucun exclu) alors quel que soit le sous-ensemble $E_{2}$, le coût $C_{12}$ ( $E_{1}$ et $E_{2}$ exclus) est supérieur au coût $C_{2}\left(E_{2}\right.$ exclu seul $)$ :

$$
C_{1}>C_{0} \Rightarrow C_{12}>C_{2}, \quad \forall E_{2} .
$$

En effet si $C_{1}>C_{0}$, il vient :

$$
F_{0}-F_{1}+P_{1}>F_{0}+P_{0}
$$

soit :

$$
P_{1}-P_{0}>F_{1} \text {. }
$$

Portons cette inégalité dans l'expression de $C_{12}$ :

$$
C_{12}=F_{0}-F_{1}-F_{2}+P_{12}>F_{0}-\left(P_{1}-P_{0}\right)-F_{2}+P_{12}
$$

D'autre part nous avons :

$$
C_{2}-P_{2}=F_{0}-F_{2} \text {. }
$$

Portons ce résultat dans l'inégalité précédente :

D'où il vient :

$$
C_{12}>C_{2}-P_{2}-\left(P_{1}-P_{0}\right)+P_{12} \text {. }
$$

$$
C_{12}-C_{2}>-P_{2}-\left(P_{1}-P_{0}\right)+P_{12}
$$

Étudions le signe du deuxième membre de cette inégalité qui peut encore s'écrire :

$$
\begin{aligned}
\sum_{j=1}^{N} \operatorname{Min}_{i \in E_{0}} u_{i j} D_{j} & +\sum_{j=1}^{N} \underset{i \in \complement_{E_{0}} E_{1} \cup E_{2}}{\operatorname{Min}} u_{i j} D_{j} \\
& -\sum_{j=1}^{N} \underset{i \in \complement_{E_{0}} E_{1}}{\operatorname{Min}} u_{i j} D_{j}-\sum_{j=1}^{N} \underset{i \in \complement_{E_{0}} E_{2}}{\operatorname{Min}} u_{i j} D_{j}
\end{aligned}
$$

Or cette expression ne peut être que positive ou nulle. En effet, pour un client $j$, trois cas sont à envisager :

1) le $u_{i j}$ minimal correspond à un établissement $i$ qui n'appartient pas à l'ensemble $E_{1} \cup E_{2}$. Alors les quatre minimums sont identiques et

$$
C_{12}-C_{2}>0
$$

2) le $u_{i j}$ minimal correspond à un établissement $i$ qui appartient à $E_{1}$. Alors :

$$
\underset{i \in \mathcal{C}_{E_{0}} E_{2}{ }^{\cdot}}{\operatorname{Min}} u_{i j}=\underset{i \in E_{0}}{\operatorname{Min}} u_{i j}
$$

Revue Française d'Automatique, Informatique et Recherche Opérationnelle 
et l'expression considérée se réduit à :

$$
\underset{i \in \complement_{E_{0}} E_{1} \cup E_{2}}{\operatorname{Min}} u_{i j}-\operatorname{Min}_{i \circ \complement_{E_{0}} E_{1}} u_{i j} .
$$

Deux cas peuvent alors se présenter :

a) le $u_{i j}$ minimal de $\oint_{E_{0}} E_{1}$ correspond à un établissement qui appartient à $\complement_{E_{0}} E_{1} \cup E_{2}$, alors l'expression est nulle;

b) le $u_{i j}$ minimal correspond à un établissement qui appartient à $E_{2}$, l'expression du second membre de l'inégalité est positive, car :

$$
\underset{i \in \complement_{E_{0}} E_{1} \cup E_{2}}{\operatorname{Min}} u_{i j}>\underset{i \in \mathfrak{C}_{E_{0}} E_{1}}{\operatorname{Min}} u_{i j} ;
$$

3) le $u_{i j}$ minimal (dans $E_{0}$ ) correspond à un établissement $i$ qui appartient à $E_{2}$. Alors pour les mêmes raisons que précédemment dans le cas où le $u_{i j}$ minimal correspondait à un établissement de $E_{1}$, l'expression ne peut être que positive ou nulle.

Par conséquent, il vient dans tous les cas :

$$
C_{1}>C_{0} \Rightarrow C_{12}-C_{2}>0, \quad \forall E_{2} \text {. }
$$

\section{Utilisation de la propriété}

L'étude systématique du problème peut être menée en excluant de l'ensemble des établissements potentiels, toutes les combinaisons de $k$ établissements que l'on peut former $(k=1, \ldots, M)$.

Les combinaisons de $k$ établissements peuvent être formées en prenant chacune des combinaisons de rang $(k-1)$ et en lui ajoutant un établissement dont le numéro d'ordre est supérieur au numéro le plus élevé figurant dans la combinaison de $(k-1)$ établissements. Cette procédure de formation est illustrée sur la figure ci-dessous :

$$
\begin{aligned}
& \text { (1) } \rightarrow(1,2) \rightarrow(1,2,3) \rightarrow \ldots \\
& \longrightarrow(1,2,4) \rightarrow \ldots \\
& \longrightarrow \ldots . . . \\
& L(1,3) \rightarrow(1,3,4) \rightarrow \ldots \\
& \llcorner(1,4) \rightarrow \ldots \ldots . \\
& (2) \rightarrow(2,3) \rightarrow \ldots \ldots \ldots
\end{aligned}
$$

Formation des combinaisons

$n^{\circ}$ octobre 1975, V-3. 
Si, au cours de l'étude des différentes combinaisons, il en apparaît une $(1,2,4)$ par exemple, dont le coût minimal associé est supérieur à celui de la combinaison qui lui a donné naissance $(1,2)$ dans notre exemple, alors en raison de la propriété précédente, les combinaisons formées à partir de celle-là $(1,2,4)$ ne peuvent pas être optimales. En effet, pour chacune d'entre elles [1, 2, $4(x)]$, il existe une combinaison [1,2(x)] présentant un coût minimal associé inférieur.

La méthode retenue pour former les combinaisons crée une filiation linéaire entre celles-ci qui n'est pas unique. En fait, chaque combinaison de $k$ établissements peut être formée à partir de $k$ combinaisons de $(k-1)$ établissements. Si le calcul est conduit de manière à ce que les coûts minimaux correspondant à toutes les combinaisons de $(k-1)$ établissements soient connus et mémorisés, il est possible de comparer le coût de chaque combinaison de $k$ établissements avec chacun des coûts associés aux combinaisons de $(k-1)$ établissements à partir desquelles elle peut être formée.

\section{SECONDE MÉTHODE}

Elle consiste à introduire une procédure de " tâtonnement » idéalisant le fonctionnement d'un marché fictif correspondant au problème étudié. Sur ce marché, les établissements et les clients sont considérés comme des agents autonomes, respectivement " offreurs " et "demandeurs ». La concurrence parfaite, au sens de la théorie économique, entre des producteurs indépendants, conduit, sous certaines hypothèses, à la production au coût minimal [10]. Une de ces hypothèses (coût de production moyen non décroissant), n'est pas satisfaite dans les conditions du problème. Nous sommes, cependant, parvenus à mettre au point une procédure de contrat entre offreurs et demandeurs et un comportement des offreurs qui conduisent à une production et une distribution à un coût très voisin du coût minimal ou même identique.

Chaque période, ou marché, de la procédure qui nous a donné les résultats les plus satisfaisants comprend deux étapes :

\section{$\mathbb{1}^{\text {re étape }}$}

Chaque offreur propose un prix, départ-usine, valable pour tous les demandeurs et déterminé selon un calcul expliqué plus loin (p. 49). Chaque demandeur choisit l'offreur le moins disant, compte tenu des différents coûts de transport. Une fois que les demandeurs ont fait leur choix, les offreurs peuvent se trouver dans l'une des trois situations suivantes :

a) aucune demande;

b) les recettes correspondant aux demandes excèdent les coûts de production;

c) les recettes correspondant aux demandes sont inférieures aux coûts de production. 


\section{$2^{e}$ étape}

Cette deuxième étape sert à apurer la situation précédente :

Les offreurs dans la situation a) se retirent du marché : ils ne proposent pas de prix lors de la deuxième étape du marché considéré.

Les offreurs dans la situation $b$ ) ne modifient pas leur prix.

Les offreurs dans la situation $c$ ) calculent, compte tenu des prix des rivaux, quel est pour chacun de leurs clients le prix-limite supérieur qu'ils peuvent pratiquer.

Le prix-limite supérieur est le prix au-delà duquel le client changerait de producteur.

Ils calculent, alors, s'ils peuvent récupérer leur coût de production en élevant leur prix jusqu'au prix-limite le plus faible. Ils peuvent, alors, rencontrer deux situations :

$c_{1}$ ) le prix-limite le plus faible permet de couvrir les coûts de production : ils proposent, alors, un prix situé à égale distance du prix de revient et de ce prix;

$c_{2}$ ) le prix-limite le plus faible ne permet pas de couvrir les coûts de production. Ils abandonnent le client marginal et recommencent les calculs pour le prix-limite immédiatement plus élevé jusqu'à ce que soit ils se retirent du marché pour l'étape considérée, soit ils parviennent à récupérer leurs coûts.

Ainsi, à la fin de la deuxième étape de chaque marché, les offreurs se répartissent en deux catégories seulement :

- une catégorie a) qui s'est retirée du marché, faute de demande;

- une catégorie $b$ ) qui a proposé un prix au moins égal au coût moyen et obtenu des demandes.

Le système de prix proposé à la première étape du premier marché est arbitraire; par contre, pour les marchés suivants, il est déterminé de la façon suivante :

Chaque offreur calcule, sur la base des prix pratiqués lors du marché précédent, le prix-limite inférieur des clients qui se sont adressés à ses rivaux. Le prix-limite inférieur de ces clients est le prix qui les détacherait de leur producteur précédent et les conduirait à se fournir auprès de l'offreur considéré. Il calcule, alors, si, en abaissant son prix de façon à proposer le prixlimite inférieur qui lui permet de gagner un client supplémentaire, il reste bénéficiaire. Sinon, il recommence les calculs pour un accroissement de $2,3, \ldots, k$ clients jusqu'à ce qu'il trouve :

a) aucune augmentation de la clientèle n'est possible : il propose, alors, son prix de la deuxième étape du marché précédent;

$\mathrm{n}^{\circ}$ octobre 1975, V-3. 
b) les coûts peuvent être couverts avec un accroissement minimal de $k$ clients. Il propose, alors, un prix situé à égale distance du prix de revient et du prix-limite inférieur correspondant au $k^{0}$ client.

Le comportement des offreurs que nous venons de décrire est inspiré de la stratégie de maximation des ventes ou de maximation de la part du marché. Toutefois, la procédure de révision des prix indique une mise en œuvre prudente de cette stratégie arbitrant entre un souci de révision appréciable à chaque étape et un souci d'éviter un blocage dans des situations éloignées de l'optimum [11].

En utilisant cette procédure, les marchés successifs sont caractérisés :

1) par l'ensemble des prix proposés par les offreurs;

2) par le coût de production et de transport correspondant, qui constitue précisément le critère que nous cherchons à minimer.

L'ensemble de prix suffit à déterminer le marché puisque les clients se bornent à choisir le moins disant sur la base des prix proposés. Aussi quand on rencontre pour la deuxième fois un ensemble de prix, est-on assuré de retrouver une répétition de tous les marchés intermédiaires. Il est, alors, possible de relancer la recherche de l'optimum en éliminant un groupe de producteurs tirés au hasard pendant une étape.

La mise en œuvre de cette procédure, retenue après des tentatives nombreuses testant différents types de comportement et de contrat, fait apparaître des états de la distribution comprenant l'optimum ou des états très voisins.

\section{MISE EN CEUVRE}

Les deux méthodes qui viennent d'être présentées ont été mises en œuvre sur un exemple comportant 20 établissements et 40 clients, ce qui correspond à la détermination de 800 variables de transport. Le tableau I représente la matrice des coûts de transport entre chaque établissement et chaque client. Les valeurs choisies sont les distances entre 40 villes prises au hasard dans le sud de la France. Le tableau I indique également le niveau de la demande de chaque client et celui des coûts, fixes et proportionnels, correspondant à chaque établissement.

Afin de mettre en évidence le rôle joué dans la détermination de l'optimum par le niveau relatif des coûts fixes, le problème a été traité plusieurs fois en multipliant les coûts fixes par un facteur $k$ qui a varié entre 0,2 et 3,0 . Les résultats obtenus par chacune des deux méthodes sont présentés dans le tableau II. Les résultats obtenus par la méthode rigoureuse sont représentés 


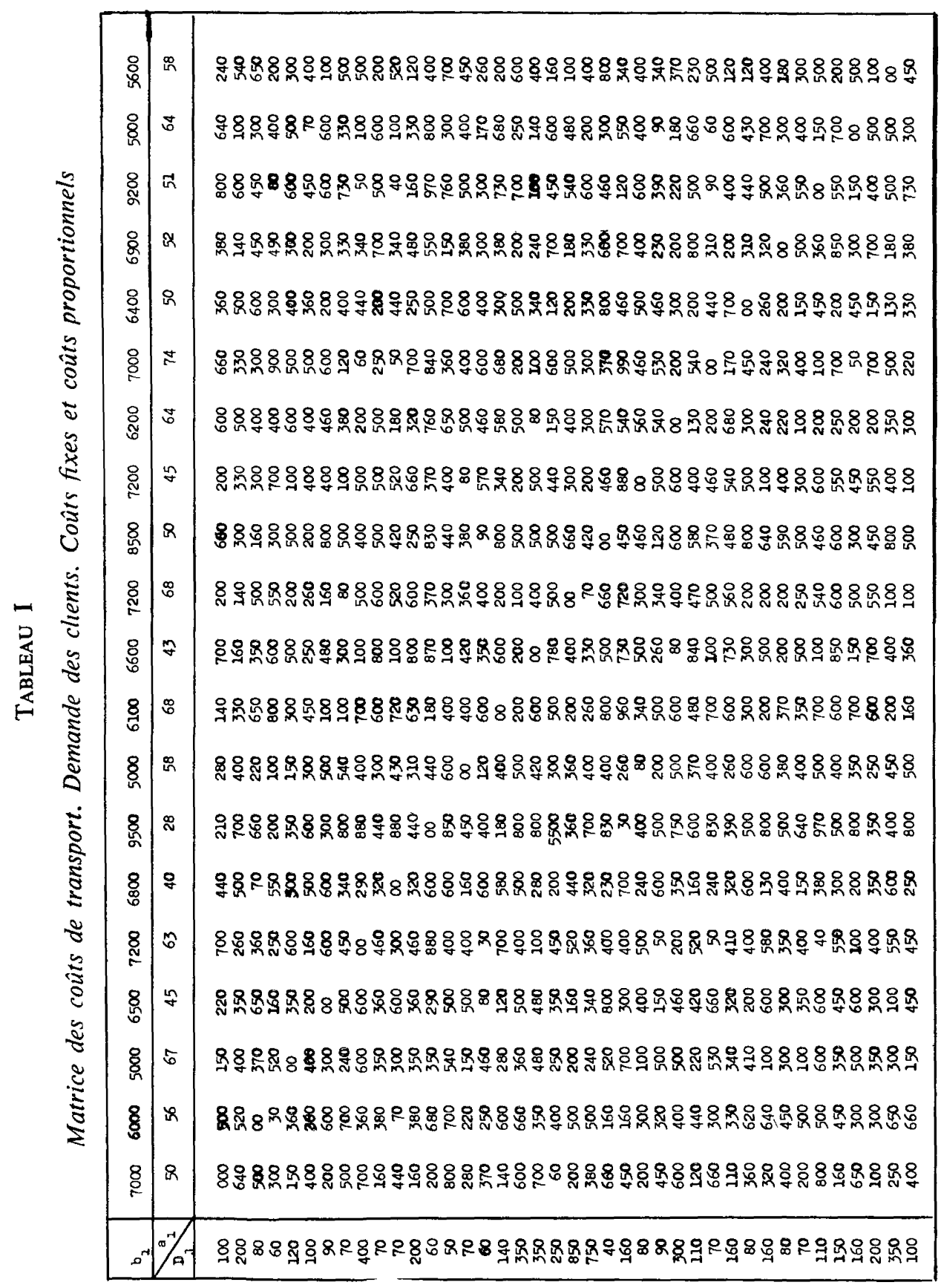

$\mathrm{n}^{\circ}$ octobre 1975, V-3. 
dans les trois colonnes de droite. La première indique le nombre d'appels à l'algorithme de minimation des coûts de transport, ce qui est une mesure du temps nécessaire à l'exécution du programme. La deuxième colonne indique le coût optimal associé à la combinaison optimale des établissements dont le nombre figure dans la troisième colonne.

\section{TABLEAU II}

\begin{tabular}{|c|c|c|c|c|c|c|c|c|c|c|c|c|c|c|c|}
\hline \multirow{3}{*}{ k } & \multicolumn{12}{|c|}{ SINULATION D'UN YARCEE } & \multirow{2}{*}{\multicolumn{3}{|c|}{$\frac{\text { ALGORITHC }}{\text { OFTIROS }}$}} \\
\hline & \multicolumn{4}{|c|}{ I } & \multicolumn{4}{|c|}{ III } & \multicolumn{4}{|c|}{ III } & & & \\
\hline & $\mathbb{N} \Delta$ & SIN & $\mathrm{NE}$ & $\%$ & NA & int & $\mathrm{NE}$ & 8 & $\mathrm{NA}$ & LEN & $\mathrm{NE}$ & $\%$ & NA & XTN & NE \\
\hline 0,2 & 10 & $7 \overline{727,5}$ & $20^{*}$ & 0 & 27 & 727,5 & $20^{*}$ & 0 & 21 & 727,5 & $20^{*}$ & 0 & 21 & 727,5 & 20 \\
\hline 0.5 & 5 & 756,9 & $19^{\prime *}$ & 0 & 18 & 767,1 & $19^{*}$ & 0,03 & 9 & 767,1 & $19^{*}$ & 0,03 & 22 & 766,9 & 19 \\
\hline 1,0 & 9 & 825,2 & 17 & 0,15 & 13 & 825,2 & 17 & 0,16 & 15 & 825,2 & 17 & 0,16 & 47 & 823,9 & 15 \\
\hline 1,5 & 10 & 872,8 & $15^{*}$ & 0,02 & 20 & 873.3 & 14 & 0,08 & 33 & 872,8 & $15^{*}$ & 0,02 & 60 & 872,6 & 15 \\
\hline 2,0 & 17 & 917,3 & 13 & 0,20 & 33 & 917,6 & 13 & 0,23 & 59 & 916,7 & $12^{*}$ & 0,14 & 549 & 915,3 & 12 \\
\hline 3,0 & 48 & 997,7 & 12 & 1,0 & 26 & 995,8 & $20^{*}$ & 0,80 & 43 & 1015 & 11 & 2,7 & 7490 & 987,8 & 10 \\
\hline
\end{tabular}

$k \quad$ : facteur multiplicatif des coûts fixes.

NA : nombre d'appels pour obtenir le coût minimal.

MIN : 1/1000 (coût de production + coût de transport) (valeur minimale obtenu par la procédure).

$\% \quad$ : écart relatif entre le coût d'approvisionnement déterminé par la procédure de marché et le coût déterminé par la méthode rigoureuse.

NE : nombre d'établissements figurant dans la solution optimale.

L'astérisque correspond au cas où les deux procédures ont donné les mêmes résultats. (Dans tous les cas, ce sont les mêmes établissements qui ont été éliminés par les deux procédures, mais il est arrivé qu'une des procédures élimine un établissement ou deux de plus que l'autre.) Les coûts minimaux diffèrent parfois pour le même nombre d'établissements éliminés parce que compte tenu des prix pratiqués dans la simulation de marché les couplages établissements-clients ne sont pas nécessairement identiques à ceux de la solution rigoureuse.

I, II, III correspondent à trois mises en œuvre de la procédure de simulation de marché avec des prix initiaux différents. 
Les résultats obtenus par la procédure de marché sont présentés dans les colonnes I, II et III. En raison de l'influence du vecteur prix initial qui est arbitraire, on a procédé à trois recherches successives de l'optimum. La première correspond à un vecteur prix initial dans lequel le prix $p_{i}$ proposé par l'établissement-offreur $i$ est déterminé de la façon suivante :

$$
p_{i}=a_{i}+b_{i} / q_{i}^{0}, \quad \forall i, \quad i=1,2, \ldots, M
$$

où $q_{i}^{0}$ correspond à une répartition du marché en parts égales :

$$
q_{i}^{0}=\left(\sum_{j=1}^{N} D_{j}\right) / M
$$

Les deux autres recherches (II et III) partent d'un vecteur prix initial totalement aléatoire.

Pour chaque recherche, le tableau II présente :

- le nombre d'appels à la procédure de confrontation des établissementsoffreurs et des clients-demandeurs;

- le coût minimal de l'approvisionnement des clients, c'est-à-dire la somme des coûts de production des offreurs " bénéficiaires 》 et des coûts de transport vers les demandeurs;

- le nombre des offreurs " bénéficiaires » correspondant au coût d'approvisionnement minimal;

- l'écart relatif entre le coût d'approvisionnement minimal et le coût correspondant à l'état optimal de la distribution et de la production déterminé par la méthode rigoureuse.

Les cas où les deux méthodes ont conduit à conserver le même ensemble d'établissements sont indiqués par une astérisque à côté du nombre d'établissements en activité à l'optimum.

\section{Discussion des résultats}

Ces résultats montrent que le nombre d'appel à l'algorithme de minimation des coûts de transports augmente avec le nombre d'établissements à éliminer. Cette augmentation est beaucoup plus rapide lorsque la méthode rigoureuse est utilisée que dans la procédure de marché. Le tableau III montre l'évolution du nombre des appels dans chaque cas.

$n^{\circ}$ octobre 1975, V-3. 


\section{Tableau III}

Comparaison des temps de calcul

\begin{tabular}{|c|c|c|c|c|c|c|}
\hline k & 0,2 & 0,5 & 1,0 & 1,5 & 2,0 & 3,0 \\
\hline $\begin{array}{c}\text { N. A. } \\
\text { (marché) }\end{array}$ & 19 & 11 & 12 & 21 & 36 & 39 \\
\hline $\begin{array}{c}\text { N. A. } \\
\text { (opt_mua) }\end{array}$ & 21 & 22 & 47 & 60 & 349 & 7490 \\
\hline
\end{tabular}

L'augmentation exponentielle du nombre des appels rend la méthode rigoureuse inutilisable quand le nombre des établissements à éliminer devient trop grand. En effet, s'il y a $n$ établissements éliminés dans la solution optimale le nombre des appels est au moins de $2^{n}$.

Ces résultats montrent, également, que la procédure de marché donne des résultats très proches de l'optimum. En raison de sa relative rapidité, elle peut être mise en œuvre plusieurs fois à partir de conditions initiales différentes ce qui augmente la probabilité de passer près de la solution optimale. De plus en comparant la liste des offreurs rejetés à chaque mise en œuvre, il est possible de déterminer ceux qu'il est le moins intéressant de conserver.

\section{CONCLUSION}

La comparaison des avantages et des défauts des deux méthodes montre que celles-ci sont complémentaires et qu'elles peuvent être utilisées séquentiellement. La procédure heuristique de simulation du marché ayant éliminé rapidement les établissements les moins efficaces, on peut utiliser l'algorithme sur un nombre d'établissements réduit, pour calculer la localisation optimale.

Pour rendre ces méthodes plus utiles dans la pratique, il serait nécessaire de modifier certaines hypothèses pour étudier les deux cas suivants :

(i) certains établissements existent déjà et leur capacité de production est limitée;

(ii) la fonction de coût présente plus d'une discontinuité.

\section{BIBLIOGRAPHIE}

[1] D. Patinkin, Note on the allocation of output, Quater. J. Econ. aout 1947, p. 651-657 et Multiple-plant firms, cartels and imperfect Competition, Quater. J. Econ., février 1947, p. 173-205.

[2] LeONTIEF VAssiLY, Multiple-plant firms: comment, Quater. J. Econ., aout 1947, p. 650-651.

Revue Française d'Automatique, Informatique et Recherche Opérationnelle 
[3] G. Hadley, Linear programming, Addison-Wesley, Reading, Mass., 1962.

[4] A. VASZONY, Scientific programming in business and industry, John Wiley, New York, 1958.

[5] F. L. HiтcHсоск, The distribution of a product from several sources to numerous localities, J. Math. Phys., 1941, p. 224-250.

[6] J. F. SharP, J. C. SNYder et J. H. GReEne, A decomposition algorithm for solving the multifacility production-transportation problem with non-linear production costs, Econometrica, Mai 1970, p. 490-506.

[7] G. B. Dantzig et W. M. HiRsch, The fixed charge problem, Research Memorandum, the Rand Corporation, $1^{\text {er }}$ décembre 1954; W. M. HiRsCH et A. J. HoffmaN, Extreme varieties, concave functions, and the fixed charge problem, Comm. pure appl. Math., 1961, p. 355-369.

[8] R. E. Bellman et S. E. Dreyfus, La programmation dynamique et ses applications, traduit de l'américain, Dunod, Paris, 1965.

[9] V. L. Smith, Investment and production, Harvard University Press, Cambridge, Mass., 1961.

[10] E. Malinvaud, Leçons de théorie microéconomique, Dunod, Paris, 1969.

[11] E. Malinvaud, Notes sur l'étude des procédures de planification, Rev. canad. d'économique, février 1968. 